\title{
Finite-Element Homogenization of Laminated Iron Cores with Inclusion of Net Circulating Currents due to Imperfect Insulation
}

\author{
Johan Gyselinck $^{1}$, Patrick Dular ${ }^{2}$, Laurent Krähenbühl ${ }^{3}$, Ruth V. Sabariego ${ }^{4}$ \\ ${ }^{1}$ BEAMS Department, Université Libre de Bruxelles (ULB), Belgium \\ ${ }^{2} \mathrm{ACE}$, Dept. of Electrical Engineering and Computer Science, University of Liège (ULg), Belgium \\ ${ }^{3}$ Université de Lyon, Ampere (CNRS UMR5005, Ecole Centrale de Lyon, INSA-Lyon, UCBL), France \\ ${ }^{4}$ Dept. of Electrical Engineering (ESAT), EnergyVille, KU Leuven, Belgium
}

\begin{abstract}
This paper deals with the time-domain homogenization of laminated cores in 2D or 3D finite element (FE) models of electromagnetic devices, allowing for net circulating current in the laminations (e.g. due to imperfect or damaged insulation). The homogenization is based on the expansion of the induction throughout the lamination thickness using a set of orthogonal polynomial basis functions, in conjunction with the magnetic vector potential (MVP) formulation. These basis functions allow for net flux, net current and skin effect in the laminations; by choosing the number of basis functions, one can compromise between accuracy and computational cost. The approach is validated through a linear 2D test case with in-plane imposed and induced current density; a brute-force model in which all laminations are finely meshed produces the reference solution. The extension to nonlinear 3D problems is expected to be straightforward.
\end{abstract}

Index Terms—Eddy currents, finite-element methods, homogenization, lamination stack.

\section{INTRODUCTION}

W HEN MODELLING electromagnetic devices comprising laminated iron cores by means of the FE method, it may be essential that the induced eddy currents, the associated losses and the ensuing skin effect in the laminations are directly considered in the resolution, and not just in the postprocessing stage. For real-life applications it is mostly prohibitive to model and discretise each lamination separately. Dedicated numerical techniques are therefore of great practical interest.

The approaches proposed in literature so far for dealing efficiently with laminated cores are applicable in the frequency and/or time domain, consider saturation (possibly hysteresis) or not, may include perpendicularly incident flux, and consist of single-step or two-step algorithms [1]-[5]. Mostly perfect insulation of the laminations is assumed, i.e. the induced current density cancels in any cross-section of a lamination. In practice net circulating current may occur due to interlamination insulation damage [6], [7], [8].

In this paper the net-current feature is added to the nonlinear time-domain homogenization method proposed in [3], [4]. The procedure is similar to the thin-shell technique in [9]. For the sake of brevity and clarity, validation is done through a $2 \mathrm{D}$ test case, with in-plane current density and out-of-plane flux density.

\section{1D LAMINATION MODEL}

Let us consider a lamination of thickness $d(-d / 2 \leq$ $z \leq d / 2$ ), homogeneous isotropic material with a constant conductivity $\sigma$ (resistivity $\rho=\sigma^{-1}$ ) and constant permeability $\mu$ (reluctivity $\nu=\mu^{-1}$ ). The flux density $b(z, t)$ and magnetic

Manuscript received July 6, 2015; revised July 28, 2015; accepted August 15, 2015. Date of current version July 29, 2014. Corresponding author: J. Gyselinck (e-mail: johan.gyselinck@ulb.ac.be).

Color versions of one or more of the figures in this paper are available online at http://ieeexplore.iee.org.

Digital Object Identifier (inserted by IEEE) field $h(z, t)=\nu b(z, t)$ are assumed along the $x$-axis, e.g., and the current density $j(z, t)$ and electric field $e(z, t)=\rho j(z, t)$ along the $y$-axis. The $1 \mathrm{D}$ eddy-current problem is governed by:

$$
\begin{gathered}
\partial_{z} h(z, t)=j(z, t), \quad \partial_{z} e(z, t)=\partial_{t} b(z, t), \quad j=\sigma e \\
\Rightarrow \quad \partial_{z}^{2} h(z, t)=\sigma \partial_{t} b(z, t), \quad \text { with } \quad h=\nu b .
\end{gathered}
$$

Relevant to the homogenization are the flux density and the current density averaged over the thickness, $b_{0}(t)=$ $\frac{1}{d} \int_{-d / 2}^{d / 2} b(z, t) \mathrm{d} z, j_{0}(t)=\frac{1}{d} \int_{-d / 2}^{d / 2} j(z, t) \mathrm{d} z$, respectively, the upper/lower/average surface magnetic field $h_{\mathrm{s}}^{ \pm}(t)=$ $h( \pm d / 2, t), h_{\mathrm{s}}=\left(h_{\mathrm{s}}^{+}+h_{\mathrm{s}}^{-}\right) / 2$, resp., and surface electric fields $e_{\mathrm{s}}^{ \pm}(t)=e( \pm d / 2, t)$ and $e_{\mathrm{s}}=\left(e_{\mathrm{s}}^{+}+e_{\mathrm{s}}^{-}\right) / 2$, resp.

Regarding the symmetry of $b, h, j$ and $e$ with respect to the middle of the lamination, $z=0$, two particular dual cases can be distinguished, namely with net flux but zero net current $\left(b(z, t)=b(-z, t), h_{\mathrm{s}}^{+}=h_{\mathrm{s}}^{-}, j(z, t)=-j(-z, t), j_{0}=0\right.$ and $\left.e_{\mathrm{S}}=0\right)$, and with net current but zero net flux $(j(z, t)=$ $j(-z, t), e_{\mathrm{s}}^{+}=e_{\mathrm{s}}^{-}, b(z, t)=-b(-z, t), b_{0}=0$ and $\left.h_{\mathrm{s}}=0\right)$, as shown in Fig. 1. (Both cases are generally present, furthermore along the two directions of the lamination, which makes the superposition of four cases.)

Denoting all complex quantities in bold (including $\boldsymbol{J}=$ $\sqrt{-1}$ ), the analytical frequency-domain solution of $(1)(2)$, at frequency $f$ and pulsation $\omega=2 \pi f$, yields the following expressions of the complex lamination-level reluctivity $\boldsymbol{\nu}$ and resistivity $\rho$ for the net-flux and net-current case respectively:

$$
\boldsymbol{\nu}=\frac{\boldsymbol{h}_{\mathrm{s}}}{\boldsymbol{b}_{0}}=\nu \boldsymbol{\Gamma}\left(d^{*}\right) \quad \text { and } \quad \boldsymbol{\rho}=\frac{\boldsymbol{e}_{\mathrm{s}}}{\boldsymbol{j}_{0}}=\rho \boldsymbol{\Gamma}\left(d^{*}\right),
$$

with the frequency dependence contained in $\boldsymbol{\Gamma}\left(d^{*}\right)$

$$
\boldsymbol{\Gamma}\left(d^{*}\right)=\frac{1+\boldsymbol{J}}{2} d^{*} \operatorname{cotanh}\left(\frac{1+\boldsymbol{J}}{2} d^{*}\right),
$$

where $d^{*}=d / \delta$ is the relative lamination thickness (with $\delta=$ $\sqrt{2 /(\omega \mu \sigma)}$ the penetration depth). 


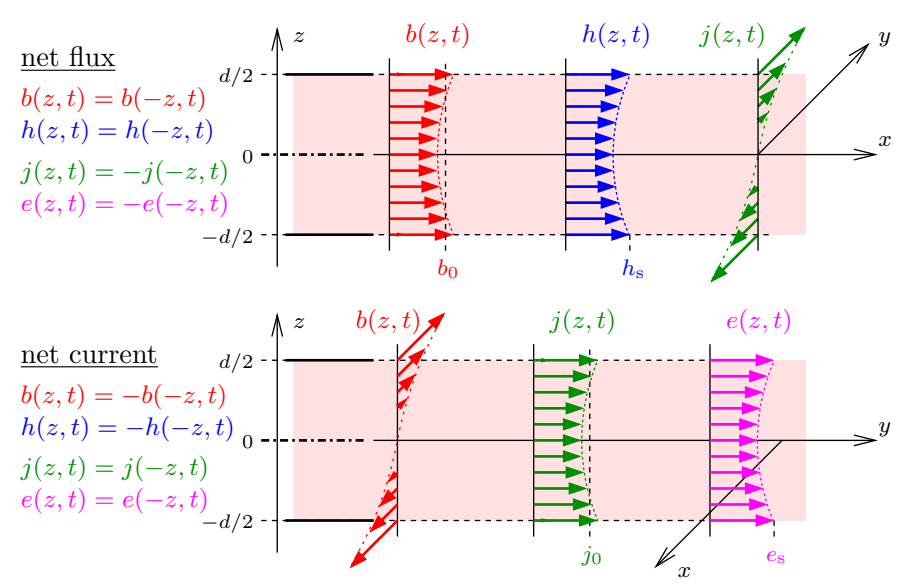

Fig. 1. 1D lamination model with local coordinate system $x y z$ and with either net flux (along $x$ ) or net current (along $y$ )

An approximate time-domain solution of (1), (2) can be obtained through expansion of $b(z, t), h(z, t)$ and $j(z, t)$ with respective polynomial basis functions (BFs) $\alpha_{k}(z), \beta_{k}(z)$ and $\gamma_{k}(z)$ of order $k$. For given $d$ and $k$, these BFs are uniquely defined, with coefficients and properties that can be obtained/verified analytically (or symbolically). The expansion of $b(z, t)$, of order $n \geq 0$, is the starting point:

$$
b(z, t)=\sum_{k=0}^{n} \alpha_{k}(z) b_{k}(t)
$$

where the BFs $\alpha_{k}(z)$ are mutually orthogonal, i.e. $\int_{-d / 2}^{d / 2} \alpha_{k}(z) \alpha_{l}(z) \mathrm{d} z=0$ if $k \neq l$, and with $\alpha_{k}(d / 2)=1$. For $n=3: \alpha_{0}(z)=1, \alpha_{1}(z)=2 \frac{z}{d}, \alpha_{2}(z)=-\frac{1}{2}+6\left(\frac{z}{d}\right)^{2}$, $\alpha_{3}(z)=-3 \frac{z}{d}+20\left(\frac{z}{d}\right)^{3}$. Even and odd BFs contribute to the net-flux and net-current part, respectively.

In order to satisfy PDE (2a) identically, the magnetic field $h(z, t)$ is next expanded considering $h_{\mathrm{s}}(t)$ and $j_{0}(t)$ :

$$
h(z, t)=h_{\mathrm{s}}(t)+j_{0}(t) z-\sum_{k=0}^{n} \sigma d^{2} \beta_{k+2}(z) \partial_{t} b_{k},
$$

with $\beta_{k}( \pm d / 2)=0$ and $-d^{2} \partial_{z}^{2} \beta_{k+2}(z)=\alpha_{k}(z)$. For $n=3$ : $\beta_{2}(z)=\frac{1}{8}-\frac{1}{2}\left(\frac{z}{d}\right)^{2}, \beta_{3}(z)=\frac{1}{12} \frac{z}{d}-\frac{1}{3}\left(\frac{z}{d}\right)^{3}, \beta_{4}(z)=-\frac{1}{32}+$ $\frac{1}{4}\left(\frac{z}{d}\right)^{2}-\frac{1}{2}\left(\frac{z}{d}\right)^{4}, \beta_{5}(z)=-\frac{1}{16} \frac{z}{d}+\frac{1}{2}\left(\frac{z}{d}\right)^{3}-\left(\frac{z}{d}\right)^{5}$.

Deriving (6) with respect to $z$ produces an expansion of $j(z, t)$ which satisfies identically PDE (1a):

$$
j(z, t)=j_{0}(t)-\sum_{k=0}^{n} \sigma d \gamma_{k+1}(z) \partial_{t} b_{k}
$$

with $\gamma_{k+1}(z)=d \partial_{z} \beta_{k+2}$. For $n=3: \gamma_{1}(z)=-\left(\frac{z}{d}\right), \gamma_{2}(z)=$ $\frac{1}{12}-\left(\frac{z}{d}\right)^{2}, \gamma_{3}(z)=\frac{1}{2} \frac{z}{d}-2\left(\frac{z}{d}\right)^{3}, \gamma_{4}(z)=-\frac{1}{16}+\frac{3}{2}\left(\frac{z}{d}\right)^{2}-$ $5\left(\frac{z}{d}\right)^{4}$.

With these three expansions all equations in (1-2) are satisfied exactly except the constitutive law $h=\nu b$. The latter can be weakly imposed by weighing with $\alpha_{l}(z)(0 \leq l \leq n)$ :

$$
\frac{1}{d} \int_{-d / 2}^{d / 2}(h(z, t)-\nu b(z, t)) \alpha_{l}(z) \mathrm{d} z=0,
$$

which leads to $n+1$ linear first-order ODEs in terms of the $n+1 b_{k}(t)$.
As the $\alpha_{k}(z)$ BFs are orthogonal and as $\beta_{k}(z)$ and $\alpha_{l}(z)$ are orthogonal unless $|k-l|$ is equal to 0 or 2 , these ODEs can be greatly simplified, with up to 4 terms per ODE.

Weighing with $\alpha_{0}(z)=1$, we obtain:

$$
h_{\mathrm{s}}=\nu b_{0}+\frac{\sigma d^{2}}{12} \partial_{t} b_{0}-\frac{\sigma d^{2}}{60} \partial_{t} b_{2},
$$

where the $\partial_{t} b_{2}$ term is to be ignored if $n<2$.

Weighing with $\alpha_{1}(z)=2 z / d$ and for any $n \geq 3$ :

$$
j_{0} \frac{d}{6}=\frac{\nu}{3} b_{1}+\frac{\sigma d^{2}}{180} \partial_{t} b_{1}-\frac{\sigma d^{2}}{420} \partial_{t} b_{3} .
$$

If $n \geq 2$, weighing with $\alpha_{l}(z), 2 \leq l \leq n$, produces $n-1$ ODEs:

$$
0=\frac{\nu}{p_{l}} b_{l}+\frac{\sigma d^{2}}{q_{l, l}} \partial_{t} b_{l}+\frac{\sigma d^{2}}{q_{l-2, l}} \partial_{t} b_{l-2}+\frac{\sigma d^{2}}{q_{l+2, l}} \partial_{t} b_{l+2},
$$

where the $\partial_{t} b_{n+1}$ and $\partial_{t} b_{n+2}$ terms are to be ignored when $l=n-1$ and $l=n$, resp., and where the $p_{l}$ and $q_{k, l}$ coefficients are given by:

$$
\begin{aligned}
& p_{l}^{-1}=\frac{1}{d} \int_{-d / 2}^{d / 2} \alpha_{l}^{2}(z) \mathrm{d} z=\frac{1}{2 l+1}, \\
& q_{k, l}^{-1}=\frac{1}{d} \int_{-d / 2}^{d / 2} \beta_{k+2}(z) \alpha_{l}(z) \mathrm{d} z .
\end{aligned}
$$

with $q_{l, l}$ equal to $12,180,210,630,1386, \ldots$, and $-q_{l+2, l}=$ $-q_{l, l+2}$ equal to $60,420,1260,2772,5148, \ldots$, for $l=$ $0,1,2,3,4, \ldots$

The average surface electric field is given by $(n \geq 1)$ :

$$
e_{\mathrm{s}}(t)=\frac{\rho}{2}(j(d / 2, t)+j(-d / 2, t))=\rho j_{0}(t)+\frac{d}{6} \partial_{t} b_{1} .
$$

Combining (10) and (14), for any $n \geq 3$, leads to

$$
e_{\mathrm{s}}=\frac{2 \nu}{\sigma d} b_{1}+\frac{d}{5} \partial_{t} b_{1}-\frac{d}{70} \partial_{t} b_{3}
$$

such that $h_{\mathrm{s}}$ and $e_{\mathrm{S}}$ can be directly used as source quantities for the $n+1$ ODEs. Solving the latter in the frequency domain $\left(\partial_{t} \equiv \jmath \omega\right)$ with either net flux $\left(\boldsymbol{h}_{\mathrm{s}}=1\right.$ and $\left.\boldsymbol{e}_{\mathrm{s}}=0\right)$ or net current ( $\boldsymbol{h}_{\mathrm{s}}=0$ and $\boldsymbol{e}_{\mathrm{s}}=1$ ), the quick convergence towards the analytical solution (3) can be observed. Roughly, allowing for a $1 \%$ error, $n$ equal to 1,3 and 5 suffices until $d^{*}$ equal to 1,4 and 8 respectively.

\section{2D FINITE ELEMENT IMPLEMENTATION}

In order to validate the 1D lamination model of the previous section, we consider a 2D magnetodynamic problem in domain $\Omega \in \mathbb{R}^{2}$ with in-plane current density $\underline{j}(x, y, t)$ (zero $z$ component) and out-of-plane flux density $\underline{b}(\bar{x}, y, t)$ (zero $x$ and $y$ component).

\section{A. Reference brute-force modelling}

Using the (in-plane) magnetic vector potential $\underline{a}$, with $\underline{b}=$ curl $\underline{a}$ and $\underline{e}=-\partial_{t} \underline{a}$, the well-known weak form of Ampere's law $\operatorname{curl} \underline{h}=\underline{j}$, with $\underline{j}=\underline{j}_{\mathrm{s}}-\sigma \partial_{t} \underline{a}$, reads: find $\underline{a}$ so that

$$
\left(\underline{j}_{\mathrm{s}}, \underline{a}^{\prime}\right)_{\Omega_{s}}=\left(\underline{h}(\underline{b}), \operatorname{curl} \underline{a}^{\prime}\right)_{\Omega}+\left(\sigma \partial_{t} \underline{a}, \underline{a}^{\prime}\right)_{\Omega_{\mathrm{c}} \cup \Omega_{\ell}}, \quad \forall \underline{a}^{\prime}
$$


where $\underline{a}^{\prime}$ is a test function; $\underline{j}_{\mathrm{s}}$ is the prescribed source current density in $\Omega_{\mathrm{s}} ;-\sigma \partial_{t} \underline{a}$ is the induced current density in the non-laminated and laminated conducting subregions $\Omega_{\mathrm{c}}$ and $\Omega_{\ell}$, resp.; $(\cdot, \cdot)_{\Omega}$ denotes a surface integral in $\Omega$ of the scalar product of the two arguments. For the sake of conciseness boundary terms are omitted in (16) and infinitely thin insulation layers are assumed between the laminations. Net currents in the laminations are therefore only possible through their lateral sides (of width $d$ ).

The basic formulation (16) requires that each lamination is modelled explicitly and meshed sufficiently finely in the thickness direction. The MVP $\underline{a}$ can be discretised with Whitney edge elements, with tree-cotree gauging in the non-conducting part of $\Omega[3]$.

\section{B. Homogenization of the lamination stack}

The homogenization of the lamination stack consists in meshing $\Omega_{\ell}$ coarsely, independently of the lamination thickness and orientation, and defining in it new variables $\underline{b}_{k}(x, y, t)$ $(1 \leq k \leq n)$ along $z$, with BFs which are, e.g., elementwise nonzero and equal to the unit vector along $z$. The average flux density in the laminations can be considered through the usual MVP, i.e. $\underline{b}_{0}(x, y, t)=\operatorname{curl} \underline{a}$ in $\Omega_{\ell}$, for which edge BFs are used throughout $\Omega$.

Considering $\underline{h}_{\mathrm{S}}$ (9), instead of $\underline{h}$ [4], and $\underline{j}_{0}$ (10) in $\Omega_{\ell},(16)$ can be rewritten as (with $n=2$, e.g.):

$$
\begin{array}{r}
\left(\underline{j}_{s}, \underline{a}^{\prime}\right)_{\Omega_{s}}=\left(\nu \operatorname{curl} \underline{a}, \operatorname{curl} \underline{a}^{\prime}\right)_{\Omega}+\left(\sigma \partial_{t} \underline{a}, \underline{a}^{\prime}\right)_{\Omega_{c}} \\
+\left(\frac{\sigma d^{2}}{12} \partial_{t} \operatorname{curl} \underline{a}, \operatorname{curl} \underline{a}^{\prime}\right)_{\Omega_{\ell}}-\left(\frac{\sigma d^{2}}{60} \partial_{t} \underline{b}_{2}, \operatorname{curl} \underline{a}^{\prime}\right)_{\Omega_{\ell}} \\
-\left(\frac{2 \nu}{d} \underline{b}_{1}^{*}, \underline{a}^{\prime}\right)_{\Omega_{\ell}}-\left(\frac{\sigma d}{30} \partial_{t} \underline{b}_{1}^{*}, \underline{a}^{\prime}\right)_{\Omega_{\ell}}+\left(\frac{\sigma d}{70} \partial_{t} \underline{b}_{3}^{*}, \underline{a}^{\prime}\right)_{\Omega_{\ell}},
\end{array}
$$

where $\underline{b}_{l}^{*}$ is an in-plane vector, parallel to the laminations, obtained by a $90^{\circ}$ rotation of $\underline{b}_{l}$.

Using test functions $\underline{b}_{l}^{\prime}, 2 \leq l \leq n$, in $\Omega_{\ell}$, (11) becomes: find $\underline{b}_{l}$, so that

$$
0=\left(\frac{\nu}{p_{l}} \underline{b}_{l}, \underline{b}_{l}^{\prime}\right)_{\Omega_{\ell}}+\left(\frac{\sigma d^{2}}{q_{l, l}} \partial_{t} \underline{b}_{l}, \underline{b}_{l}^{\prime}\right)_{\Omega_{\ell}}+\ldots, \quad \forall \underline{b}_{l}^{\prime}
$$

Finally, (14) is weakly imposed with $\underline{b}_{1}^{\prime}$ :

$$
0=\left(\partial_{t} \underline{a}^{*}, \underline{b}_{1}^{\prime}\right)_{\Omega_{\ell}}+\left(\frac{2 \nu}{\sigma d} \underline{b}_{1}, \underline{b}_{1}^{\prime}\right)_{\Omega_{\ell}}+\left(\frac{d}{5} \partial_{t} \underline{b}_{1}, \underline{b}_{1}^{\prime}\right)_{\Omega_{\ell}}-\ldots
$$

with $e_{\mathrm{s}}^{*}=-\partial_{t} \underline{a}^{*}$ a $90^{\circ}$ rotation of $e_{\mathrm{s}}$.

\section{APPLiCATion EXAMPLE}

The presented homogenization approach is applied to the $2 \mathrm{D}$ model of a laminated ring core (neglecting curvature, $1 \mathrm{~m}$ along $z$ ) shown in Fig. 2 . The core comprises $2 \times 10$ laminations with $d=0.5 \mathrm{~mm}, \sigma=2 \mathrm{MS} / \mathrm{m}$, relative permeability $\mu_{\mathrm{r}}=2000$, width $2 \times 10 \mathrm{~mm}$, separated by horizontal insulation layers (70 $\mu \mathrm{m}$ thick, $\sigma=0, \mu_{\mathrm{r}}=1$ ). In the fine model (Fig. 2, left) each half lamination cross-section is split up in $13 \times 10$ quadrangles, separated by a single layer of quadrangles (insulation), for a total of 5065 real or complex valued unknowns (after gauging). The homogenized lamination stack (including the insulation) is split up in $13 \times 10$ quadrangles (Fig. 2, right), with 130 additional unknowns per $\underline{b}_{l}(1 \leq l \leq n)$, on top of the 352 when $n=0$. The fill factor of the laminated core, $\lambda=50 / 57=87.7 \%$, is simply taken into account through an increased reluctivity $\nu / \lambda$ and decreased conductivity $\lambda \sigma$ [3].
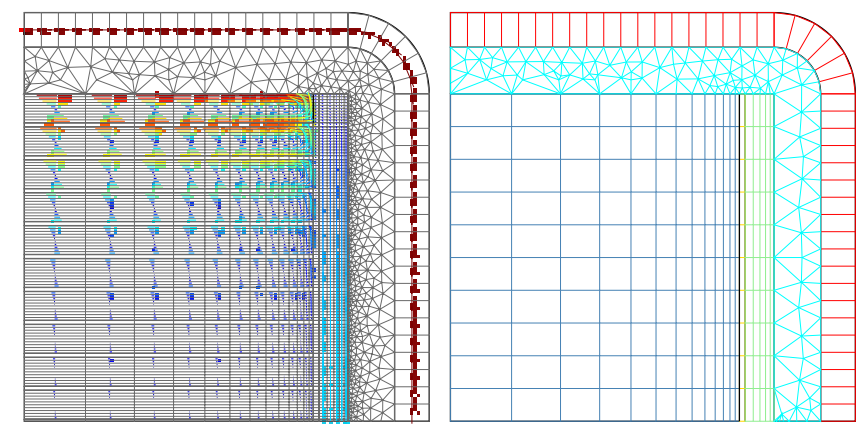

Fig. 2. 2D FE model with laminated ring core, lateral contact layers, coil and air in-between (1/4 cross-section). Left: fine reference mesh with imposed and induced current density $(2 \mathrm{kHz}$, real part); Right: mesh of homogenized lamination stack

The lamination stack touches a vertical $0.1 \mathrm{~mm}$ thick conducting layer $\left(\mu_{r}=1, \sigma=500 \mathrm{~S} / \mathrm{m}\right)$, which touches in turn a $0.5 \mathrm{~mm}$ thick conducting layer $\left(\mu_{r}=1, \sigma=5 \mathrm{MS} / \mathrm{m}\right)$. These layers constitute $\Omega_{\mathrm{c}}$ and allow for net horizontal current $\underline{j}_{0}$ in the laminations and a global skin effect in the stack.

The source current density $\underline{j}_{\mathrm{s}}$ in the coil $\left(\Omega_{\mathrm{s}}\right.$, outer layer of quadrangles) varies either sinusoidally or triangularly in time (up to $10 \mathrm{kHz}, d^{*}=d / \delta=6.3$ ) with the same peak value. The latter is such that in DC conditions the flux density in the laminations is around $1 \mathrm{~T}$. In the sinusoidal case (with imposed real-valued current), the equations are solved using complex numbers (producing the steady-state regime), whereas in the triangular case, time stepping is done starting from the zero solution (using backward Euler and 200 steps per period).

Figs. 2 (left) and 3 show results for the sinusoidal regime at $2 \mathrm{kHz}\left(d^{*}=d / \delta=2.8\right)$, with both local and global skin effect. The contribution of $b_{0}, b_{1}$ and $b_{2}$ to the total flux density is shown in more detail in Fig. 4. Clearly the homogenization with $n=2$ gives a good agreement with the reference solution.

Some time-stepping results with triangular current are shown in Fig. 5, namely total eddy current losses and magnetic energy in the laminations versus time, for different frequencies (up to $10 \mathrm{kHz}$ ), obtained with the reference model and homogenization with increasing $n$, by integrating either $j^{2}(x, y, t) / \sigma$ and $\frac{1}{2} \nu b^{2}(x, y, t)$ or the corresponding expressions in terms of $b_{k}(x, y, t)(0 \leq k \leq n)$. Given the harmonic content of the current waveform, $n=5$ is a reasonable choice at $10 \mathrm{kHz}$.

For 200 time steps, the computation time on a MacBook Pro $2.6 \mathrm{GHz}$ Quad-core Intel i7 is approximately $35 \mathrm{~s}$ with the fine mesh, and $5.7 \mathrm{~s}, 7.2 \mathrm{~s}$ and $8.3 \mathrm{~s}$ with homogenization and $n$ equal to 1,3 and 5 resp., with only a slight dependence on frequency.

\section{CONCLUSION}

The time-domain one-step homogenization approach presented in this paper is an extension of previous work by the 

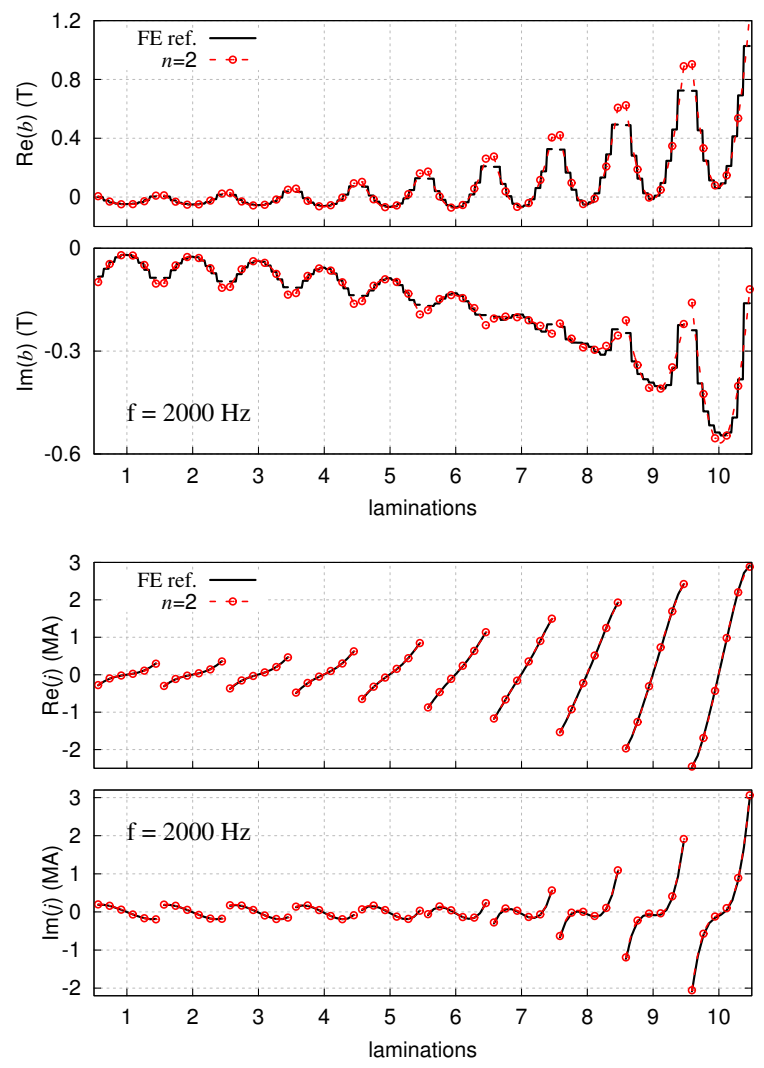

Fig. 3. Sinusoidal regime at $2 \mathrm{kHz}$ : variation of flux density and current density (real and imaginary part) throughout the ten laminations (on the vertical symmetry axis, $\underline{b}$ along $z, x$ component of $\underline{j}$ ), obtained with fine mesh (FE ref.) and with homogenization $(n=2)$
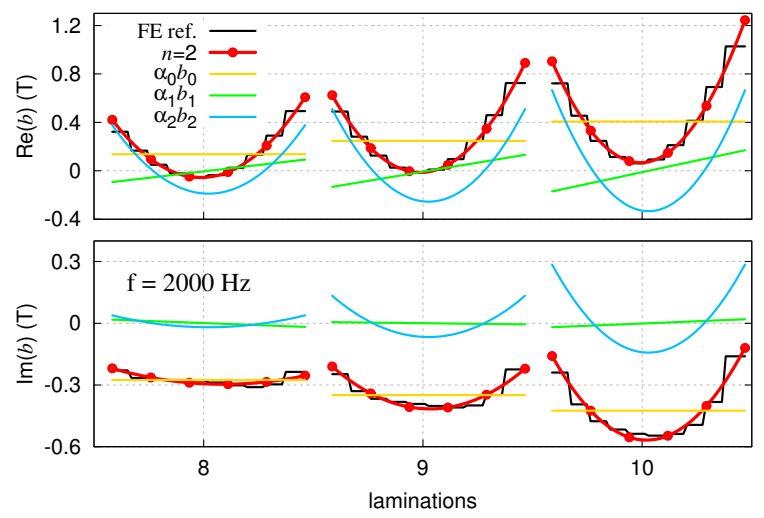

Fig. 4. Sinusoidal regime at $2 \mathrm{kHz}$ : variation of flux density throughout the three upper (outer) laminations of the core, obtained with fine mesh and homogenization $n=2$ (plus contribution of $b_{0}, b_{1}$ and $b_{2}$ )

authors as net currents in the laminations are allowed for thanks to odd basis functions for the expansion of the flux density throughout the lamination thickness. These net currents can be due to imperfect insulation and may be such that homogenization of the stack is still feasible; in the simple 2D application example the conductivity of one lateral insulation layer has been chosen sufficiently low to that end. Provided enough basis functions are adopted, both frequency and time
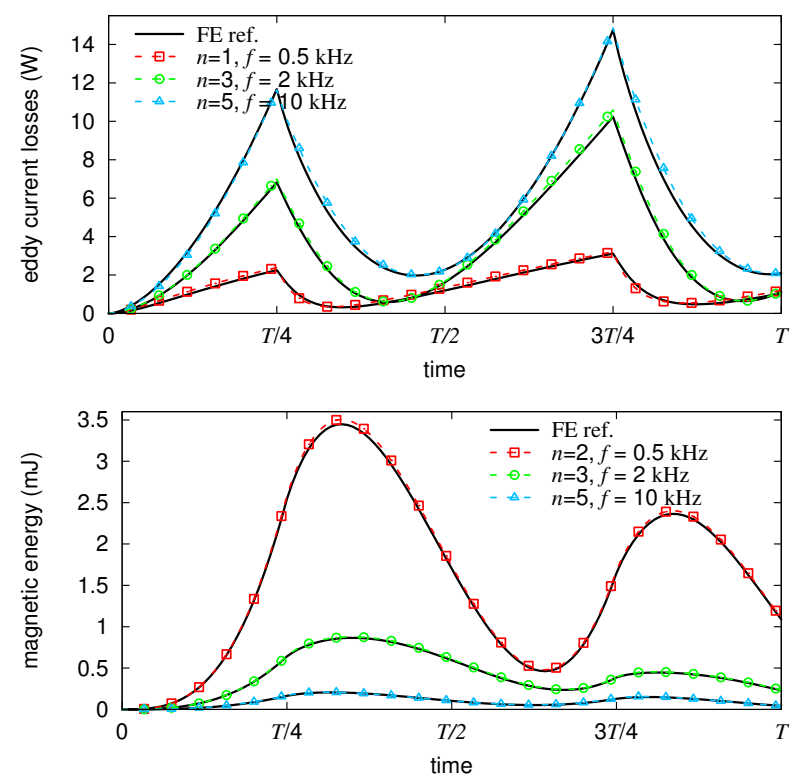

Fig. 5. Triangular current and time stepping: eddy current losses and magnetic energy versus time at $0.5,2$ and $10 \mathrm{kHz}$, obtained with fine mesh and homogenization (with increasing $n$ for sufficient accuracy)

domain results were observed to agree well with the reference solution obtained with a brute-force approach, with a reduction of the computation time. The logical next steps are to consider saturation, what is expected to be as straightforward as in [4], [9], and the application to a full 3D test case.

\section{ACKNOWLEDGEMENT}

This work was supported in part by the Walloon Region of Belgium (WBGreen FEDO, grant RW-1217703) and the Belgian Science Policy (IAP P7/02).

\section{REFERENCES}

[1] O. Bottauscio, M. Chiampi, "Analysis of laminated cores through a directly coupled 2-D/1-D electromagnetic field formulation," IEEE Trans. Magn., vol. 38, no. 5, pp. 2358-2361, 2002.

[2] J. Pippuri, A. Arkkio, "Time-harmonic induction-machine model including hysteresis and eddy currents in steel laminations," IEEE Trans. Magn., vol. 45, no. 7, pp. 2981-2989, 2009.

[3] J. Gyselinck, P. Dular, "A time-domain homogenization technique for laminated iron cores in 3D finite element model," IEEE Trans. Magn., vol. 40, no. 3, pp. 1424-1427, 2004.

[4] J. Gyselinck, R. V. Sabariego, P. Dular, "A nonlinear time-domain homogenization technique for laminated iron cores in 3-D finite-element models," IEEE Trans. Magn., vol. 42, no. 4, pp. 763-766, 2006.

[5] I. Niyonzima, R. V. Sabariego, P. Dular, F. Henrotte, C. Geuzaine, "Computational homogenization for laminated ferromagnetic cores in magnetodynamics," IEEE Trans. Magn., vol. 49, no. 5, pp. 2049-2052, 2013.

[6] D. Bertenshaw, A. C. Smith, C. W. Ho, T. Chan, M. Sasic, "Detection of stator core faults in large electrical machines," IET Electric Power Application, vol. 6, no. 6, pp. 295-301, 2012.

[7] P. Handgruber, A. Stermecki, O.Biro, G.Ofnery, "Evaluation of interlaminar eddy currents in induction machines," IECON 2013-39th Annual Conference of the IEEE, pp. 2792-2797.

[8] S. B. Shah, P. Rasilo, A. Belahcen, A. Arkkio, "Modeling of losses due to inter-laminar short-circuit currents in lamination stacks," Electrical, Control and Communication Engineering, vol. 3, no. 1, pp. 31-36, 2013.

[9] R. V. Sabariego, C. Geuzaine, P. Dular, J. Gyselinck, "Nonlinear timedomain finite-element modeling of thin electromagnetic shell," IEEE Trans. Magn., vol. 45, no. 3, pp. 976-979, 2009. 